FACTA UNIVERSITATIS (NIŠ)

Ser. Math. Inform. Vol. 34, No 5 (2019), 941-955

https://doi.org/10.22190/FUMI1905941N

\title{
SOME PROPERTIES OF EXPONENTIALLY PREINVEX FUNCTIONS
}

\author{
Muhammad Aslam Noor and Khalida Inayat Noor
}

(C) 2019 by University of Niš, Serbia | Creative Commons Licence: CC BY-NC-ND

Abstract. In this paper, we introduce some new concepts of the exponentially preinvex functions. We investigate several properties of the exponentially preinvex functions and discuss their relations with convex functions. Optimality conditions are characterized by a class of variational-like inequalities. Several interesting results characterizing the exponentially preinvex functions are obtained. Results obtained in this paper can be viewed as significant improvement of previously known results.

Keywords: preinvex function; convex function; convexity; nonlinear problems; variational inequalities.

\section{Introduction}

Convex functions and convex sets have played an important and fundamental part in the development of various fields of pure and applied sciences. Convexity theory describes a broad spectrum of very interesting developments involving a link among various fields of mathematics, physics, economics and engineering sciences. Some of these developments have made mutually enriching contacts with other fields. Ideas explaining these concepts led to the developments of new and powerful techniques to solve a wide class of linear and nonlinear problems. Convexity theory provides us with a unified framework to develop highly efficient and powerful numerical methods to solve nonlinear problems, see $[1,2,4,5,7,8,9,10,11,12,13,14,15,16$, $17,18,19,21,22,23,24,28,29,30,31,32,33]$. In recent years, various extensions and generalizations of convex functions and convex sets have been considered and studied using innovative ideas and techniques. It is known that more accurate and inequalities can be obtained using the logarithmically convex functions than the convex functions. Closely related to the log-convex functions, we the concept of exponentially convex(concave) functions, which have important applications in information theory, big data analysis, machine learning and statistic, see, for example, $[1,32]$ and the references therein. Exponentially convex(concave) functions can

Received February 15, 2019; accepted August 14, 2019

2010 Mathematics Subject Classification. Primary 26D15; Secondary 26D10, 49J40. 
be considered as a significant extension of the convex functions. Pal and Wong [32] have discussed its role in information geometry and statistics. Antczak [2] introuced these exponentially convex functions implicitly and discuss their role in mathematical programming. We would like to mention that the origin of exponentially convex functions can be traced back to Bernstein [6]. Avriel [3] introduced and studied the concept of $r$-convex functions, where as the $(r, p)$-convex functions were studied by Antczak [2]. The definition of exponential convex functions in Noor and Noor $[25,26,27,28]$ and Alirazaei and Mathur [1] is quite different from Bernstein [6] and Pecaric et al.[30, 31]. Alirazaie and Mathur [1] , Dragomir and Gomm [9, 10] and Noor and Noor $[25,26,27,28]$ have derived several results for exponentially convex functions.

Hanson [12] studied the concept of invex functions involving an arbitrary bifunction to consider the mathematical programming problems. The invex functions appeared to be a significant generalization of the convex functions. Ben-Israel and Mond [5] introduced the invex sets and preinvex functions involving the bifunction, which can be viewed as an important contribution in the field of optimization. They proved that the differentiable preinvex functions imply the invex function, but the converse is not true in general. Mohen and Neogy [14] showed that the differentiable preinvex and invex functions are equivalent under suitable conditione. Noor [16] proved that the optimality conditions of the differentiable preinvex functions can be characterized by a class of variational inequalities, which is called variational-like inequality. For the applications, formulation and other aspects of variational-like inequalities and related equilibrium like problems, see [16, 17, 18, 19, 22, 23, 24, 28]. Antczak [2] introduced an discussed the properties of the preinvex functions in nonlinear optimization and mathematical programming.

Motivated and inspired by the ongoing research in this interesting, applicable and dynamic field, we introduce the exponentially preinvex functions. It is has been shown that the exponentially convex(concave) have nice nice properties which convex functions enjoy. Several new concepts have been introduced and investigated. We show that the local minimum of the exponentially preinvex functions is the global minimum. The optimal conditions of the differentiable exponentially preinvex functions can be characterized by a class of variational inequalities, which is called exponentially variational-like inequality, which is itself an interesting outcome of our main results. The difference (sum) of the exponentially preinvex functions and exponentially affine preinvex functions is again a exponentially convex function. The ideas and techniques of this paper may be starting point for further research in these areas.

\section{Preliminary Results}

Let $K$ be a nonempty closed set in a real Hilbert space $H$. We denote by $\langle\cdot, \cdot\rangle$ and $\|\cdot\|$ by the inner product and norm, respectively. Let $F: K \rightarrow R$ be a continuous function. 
Definition 2.1. [5] The set $K_{\eta}$ in $H$ is said to be invex set, if there exists a bifunction $\eta(.,$.$) , such that$

$$
u+t \eta(v, u) \in K, \quad \forall u, v \in K_{\eta}, t \in[0,1] .
$$

Definition 2.2. [5] A function $F$ on the invex set set $K_{\eta}$ in $H$ is said to be a preinvex funtion, if there exists a bifunction $\eta(.,$.$) , such that$

$$
F(u+t \eta(v, u)) \leq(1-t) F(u)+t F(v), \quad \forall u, v \in K_{\eta}, t \in[0,1]
$$

Definition 2.3. [5] A function $F$ on the set $K_{\eta}$ in $H$ is said to be log-preinvex , if there exists a bifunction $\eta(.,$.$) , such that$

$$
F(u+t \eta(v, u)) \leq(F(u))^{1-t}(F(v))^{t}, \quad \forall u, v \in K_{\eta}, t \in[0,1] .
$$

We now define the concept of exponentially preinvex functions and their variant forms.

Definition 2.4. A function $F$ is said to be exponentially preinvex with respect to an arbitrary bifunction $\eta(v, u)$, if

$$
e^{F(u+t \eta(v, u))} \leq(1-t) e^{F(u)}+t e^{F(v)}, \quad \forall u, v \in K_{\eta}, \quad t \in[0,1] .
$$

One can easily show that Definition 2.4 is equivalent to the following definition which is mainly due to Antczak [2].

Definition 2.5. [5] A function $F$ is said to be exponentially preinvex with respect to an arbitrary bifunction $\eta(v, u)$. if

$(2.4) F(u+t \eta(v, u)) \leq \log \left\{(1-t) e^{F(u)}+t e^{F(v)}\right\}, \quad \forall u, v \in K_{\eta}, \quad t \in[0,1]$,

Definition 2.6. A function $F$ is said to be log-preinvex with respect to an arbitrary bifunction $\eta(v, u)$, if

$$
e^{F(u+t \eta(v, u))} \leq\left(e^{F(v)}\right)^{1-t}\left(e^{F(v)}\right)^{t}, \quad \forall u, v \in K_{\eta}, \quad t \in[0,1] .
$$

From definition 2.6, it follows that

$$
e^{F(u+t \eta(v, u))} \leq(1-t) e^{F(u)}+t e^{F(v)}, \quad \forall u, v \in K_{\eta}, \quad t \in[0,1] .
$$

For $t=1$, the Definition 2.4 reduces to

$$
e^{F(u+\eta(v, u))} \leq e^{F(v)}, \quad \forall u, v \in K_{\eta}, \quad t \in[0,1],
$$

which is known as Condition A.

A function is called the exponentially prencave function $F$, if $-F$ is exponentially preinvex function. For the applications of the exponentially preinvex functions in mathematical programming, see Antczak[2].

We remark that if $\eta(v, u)=v-u$, then the invex set $K_{\eta}=K$, the convex set and Definition 2.4 reduces to: 
Definition 2.7. A function $F$ is said to be exponentially convex function, if

$$
e^{F(u+t(v-u))} \leq(1-t) e^{F(u)}+t e^{F(v)}, \quad \forall u, v \in K, \quad t \in[0,1] .
$$

For the applications of the exponentially convex(concave) functions in the mathematical programming and information theory, see Antczak [2] and Alirezaei and Mathar[1].

Example 2.1. [1] The error function

$$
\operatorname{erf}(x)=\frac{2}{\sqrt{\pi}} \int_{0}^{x} e^{-t^{2}} d t
$$

becomes an exponentially concave function in the form $\operatorname{erf}(\sqrt{x}), \quad x \geq 0$, which describes the bit/symbol error probability of communication systems depending on the square root of the underlying signal-to-noise ratio. This shows that the exponentially concave functions can play important part in communication theory and information theory.

For the properties of differentiable exponential preinvex functions, see Noor and Noor [28].

Definition 2.8. The function $F$ on the convex set $K_{\eta}$ is said to be exponentially quasi preinvex with respect to an arbitrary bifunction $\eta(v, u)$, if

$$
e^{F(u+t \eta(v, u)} \leq \max \left\{e^{F(u)}, e^{F(v)}\right\}, \quad \forall u, v \in K_{\eta}, t \in[0,1] .
$$

From the above definitions, we have

$$
\begin{aligned}
e^{F(u+t \eta(v, u))} & \leq\left(e^{(F(u)}\right)^{1-t}\left(e^{F(v)}\right)^{t} \\
& \left.\leq(1-t) e^{F(u)}+t e^{F(v)}\right) \\
& \leq \max \left\{e^{F(u)}, e^{F(v)}\right\}
\end{aligned}
$$

This shows that every exponentially log-preinvex function is a exponentially convex function and every exponentially convex function is a is a exponentially quasipreinvex function. However, the converse is not true.

Let $K_{\eta}=I_{\eta}=[a, a+\eta(b, a)]$ be the interval. We now define the exponentially preinvex function on $I_{\eta}$.

Definition 2.9. Let $I_{\eta}=[a, a+\eta(b, a)]$. Then $F$ is exponentially preinvex function, if and only if,

$$
\left|\begin{array}{ccc}
1 & 1 & 1 \\
a & x & a+\eta(b, a) \\
e^{F(a)} & e^{F(x)} & e^{F(b)}
\end{array}\right| \geq 0 ; \quad a \leq x \leq a+\eta(b, a) .
$$

One can easily show that the following are equivalent: 
1. $F$ is exponentially preinvex function.

2. $e^{F(x)} \leq e^{F(a)}+\frac{e^{F(b)}-e^{F(a)}}{\eta(b, a)}(a-x)$.

3. $\frac{e^{F(x)}-e^{F(a)}}{x-a} \leq \frac{e^{F(b)}-e^{F(a)}}{\eta(b, a)}$.

4. $(a-x+\eta(b, a)) e^{F(a)}+\eta(b, a) e^{F(x)}+(x-a) e^{F(b)} \geq 0$.

where $x=a+t \eta(b, a) \in[0,1]$.

\section{Main Results}

In this section, we consider some basic properties of exponentially general preinvex functions.

Theorem 3.1. Let $F$ be a exponentially preinvex function. Then any local minimum of $F$ is a global minimum.

Proof. Let the exponentially preinvex function $F$ have a local minimum at $u \in K_{\eta}$. Assume the contrary, that is, $F(v)<F(u)$ for some $v \in K_{\eta}$. Since $F$ is exponentially preinvex, so

$$
e^{F(u+t \eta(v, u)} \leq(1-t) e^{F(u)}+t e^{F(v)}, \quad \text { for } \quad 0<t<1 .
$$

Thus

$$
e^{F(u+t \eta(v, u))}-e^{F(u)} \leq t\left[e^{F(v)}-e^{F(u)}\right]<0,
$$

from which it follows that

$$
e^{F(u+t \eta(v, u))}<e^{F(u)},
$$

for arbitrary small $t>0$, contradicting the local minimum.

Theorem 3.2. If the function $F$ on the invex set $K_{\eta}$ is exponentially preinvex, then the level set $L_{\alpha}=\left\{u \in K_{\eta}: e^{F(u)} \leq \alpha, \quad \alpha \in R\right\}$ is an invex set.

Proof. Let $u, v \in L_{\alpha}$. Then $e^{F(u)} \leq \alpha$ and $e^{F(v)} \leq \alpha$. Now, $\forall t \in(0,1), \quad v=$ $u+t \eta(v, u) \in K_{\eta}$, since $K_{\eta}$ is an invex set. Thus, by the exponentially preinvexity of $F$, we have

$$
\begin{aligned}
e^{F(u+t \eta(v, u))} & \leq(1-t) e^{F(u)}+t e^{F(v)} \\
& \leq(1-t) \alpha+t \alpha=\alpha
\end{aligned}
$$

from which it follows that $u+t \eta(v, u) \in L_{\alpha}$ Hence $L_{\alpha}$ is an invex set.

Theorem 3.3. The function $F$ is a exponentially preinvex, if and only if,

$$
\operatorname{epi}(F)=\left\{(u, a): u \in K_{\eta}: e^{F(u)} \leq \alpha, \alpha \in R\right\}
$$

is a general invex set. 
Proof. Assume that $F$ is exponentially preinvex. Let $(u, \alpha), \quad(v, \beta) \in \operatorname{epi}(F)$. Then it follows that $e^{F(u)} \leq \alpha$ and $e^{F(v)} \leq \beta$. Thus, $\forall t \in[0,1], \quad u . v \in K_{\eta}$, we have

$$
e^{F(u+t \eta(v, u))} \leq(1-t) e^{F(u)}+t e^{F(v)} \leq(1-t) \alpha+t \beta,
$$

which implies that $(u+t \eta(v, u),(1-t) \alpha+t \beta) \in e p i(F)$. Thus epi $(F)$ is an invex set. Conversely, let epi(F) be an invex set. Let $u, v \in K_{\eta}$. Then $\left(u, e^{F(u)}\right) \in \operatorname{epi}(F)$ and $\left(v, e^{F(v)}\right) \in \operatorname{epi}(F)$. Since epi(F) is an invex set, we must have

$$
\left(u+t \eta(v, u),(1-t) e^{F(u)}+t e^{F(v))} \in e p i(F),\right.
$$

which implies that

$$
e^{F(u+t \eta(v, u)} \leq(1-t) e^{F(u)}+t e^{F(v} .
$$

This shows that $\mathrm{F}$ is an exponentially preinvex function.

Theorem 3.4. The function $F$ is exponentially quasi preinvex, if and only if, the level set $L_{\alpha}=\left\{u \in K_{\eta}, \alpha \in R: e^{F(u)} \leq \alpha\right\}$ is an invex set.

Proof. Let $u, v \in L_{\alpha}$. Then $u, v \in K_{\eta}$ and $\max \left(e^{F(u)}, e^{F(v)}\right) \leq \alpha$. Now for $t \in$ $(0,1), w=u+t \eta(v, u) \in K_{\eta}$, by the invexity of $K_{\eta}$. We have to prove that $u+$ $t \eta(v, u) \in L_{\alpha}$. By the exponentially general preinvexity of $F$, we have

$$
e^{F(u+t \eta(v, u))} \leq \max \left(e^{F(u)}, e^{F(v)}\right) \leq \alpha,
$$

which implies that $u+t \eta(v, u) \in L_{\alpha}$, showing that the level set $L_{\alpha}$ is indeed a invex set.

Conversely, assume that $L_{\alpha}$ is an invex set. Then, for any $u, v \in L_{\alpha}, t \in[0,1]$, $u+t \eta(v, u) \in L_{\alpha}$. Let $u, v \in L_{\alpha}$ for $\alpha=\operatorname{maxe}^{F(u)}, e^{F(v)}$ and $e^{F(v)} \leq e^{F(u)}$. Then from the definition of the level set $L_{\alpha}$, it follows that

$$
e^{F(u+t \eta(v, u))} \leq \max \left(e^{F(u)}, e^{F(v)}\right) \leq \alpha .
$$

Thus $F$ is an exponentially quasi preinvex function. This completes the proof.

Theorem 3.5. Let $F$ be an exponentially preinvex function. Let $\mu=\inf _{u \in K_{\eta}} F(u)$. Then the set $E=\left\{u \in K_{\eta}: e^{F(u)}=\mu\right\}$ is an invex set of $K$. If $F$ is strictly exponentially preinvex, then $E$ is a singleton.

Proof. Let $u, v \in E$. For $0<t<1$, let $w=u+t \eta(v, u)$. Since $F$ is a exponentially peinvex function, then

$$
F(w)=e^{F(u+t \eta(v, u))} \leq(1-t) e^{F(u)}+t e^{F(v)}=t \mu+(1-t) \mu=\mu,
$$

which implies that to $w \in E$. and hence $E$ is an invex set. For the second part, assume to the contrary that $F(u)=F(v)=\mu$. Since $K_{\eta}$ is an innvex set, then for $0<t<1, u+t \eta(v, u) \in K_{\eta}$. Further, since $F$ is strictly exponentially preinvex function,

$$
e^{F(u+t \eta(v, u))}<(1-t) e^{F(u)}+t e^{F(v)}=(1-t) \mu+t \mu=\mu .
$$

This contradicts the fact that $\mu=\inf _{u \in K_{\eta}} F(u)$ and hence the result follows. 
Theorem 3.6. If $F$ is exponentially preinvex function such that

$$
e^{F(v)}<e^{F(u)}, \forall u, v \in K_{\eta}
$$

then $F$ is a strictly exponentially quasi preinvex function.

Proof. By the exponentially prinvexity of the function $F$, we have

$$
e^{F(u+t \eta(v, u))} \leq(1-t) e^{F(u)}+t e^{F(v)}<e^{F}(u), \forall u, v \in K_{\eta}, t \in[0,1],
$$

since $e^{F(g(v))}<e^{F(g(u))}$, which shows that the function $F$ is a strictly exponentially quasi preinvex function.

We now discuss the properties of the differentiable general preinvex functions. For this, we need the following, which is mainly due to Mohen and Neogy [14]:

Condition C. Let $\eta(.,):. K_{\eta} \times K_{\eta} \rightarrow H$ satisfy the following assumptions

$$
\begin{aligned}
\eta(u, u+t \eta(v \cdot u)) & =-t \eta(v \cdot u)), \\
\eta(v, u+t \eta(v \cdot u))) & =(1-t) \eta(v, u), \quad \forall u, v \in) K_{\eta}, t \in[0,1] .
\end{aligned}
$$

Theorem 3.7. Let $F$ be a differentiable function and Condition $C$ hold. Then the function $F$ is exponentially preinnvex function, if and only if,

$$
\left.e^{F(v)}-e^{F(u}\right) \geq\left\langle e^{F(u)} F^{\prime}(u), \eta(v \cdot u)\right\rangle, \quad \forall v, u \in K_{\eta} .
$$

Proof. Let $F$ be an exponentially preinvex function. Then

$$
e^{F(u+t \eta(v, u))} \leq(1-t) e^{F(u)}+t e^{F(v)}, \quad \forall u, v \in K_{\eta},
$$

which can be written as

$$
e^{F(v)}-e^{F(u)} \geq\left\{\frac{e^{F(u+t \eta(v, u))}-e^{F(u)}}{t}\right\} .
$$

Taking the limit in the above inequality as $t \rightarrow 0$, we have

$$
e^{F(v)}-e^{F(u)} \geq\left\langle e^{F(u)} F^{\prime}(u), \eta(v, u)\right\rangle,
$$

which is (3.1), the required result.

Conversely, let (3.1) hold. Then

$$
\forall u, v \in K_{\eta}, t \in[0,1], \quad v_{t}=u+t \eta(v, u) \in K_{\eta} .
$$

Using Condition C, we have

$$
\begin{aligned}
e^{F(v)}-e^{F\left(v_{t}\right)} & \geq\left\langle e^{F\left(v_{t}\right)} F^{\prime}\left(v_{t}\right), \eta\left(v, v_{t}\right)\right\rangle \\
& \left.=(1-t)\left\langle e^{F\left(v_{t}\right)} F^{\prime}\left(v_{t}\right), \eta(v, u)\right)\right\rangle .
\end{aligned}
$$


In a similar way, we have

$$
\begin{aligned}
e^{F(u)}-e^{F\left(v_{t}\right)} & \geq\left\langle e^{F\left(v_{t}\right)} F^{\prime}\left(v_{t}\right), \eta\left(u,, v_{t}\right)\right\rangle \\
& =-t\left\langle e^{F\left(v_{t}\right)} F^{\prime}\left(v_{t}\right), \eta(v, u)\right\rangle .
\end{aligned}
$$

Multiplying (3.2) by $t$ and (3.3) by $(1-t)$ and adding the resultant, we have

$$
e^{F(u+t \eta(v, u)} \leq(1-t) e^{F(u)}+t e^{F(v)}
$$

showing that $F$ is a exponentially preinvex function.

Remark 3.1. From (3.1), we have

$$
e^{F(v)-F(u)}-1 \geq\left\langle F^{\prime}(u), \eta(v \cdot u)\right\rangle, \quad \forall v, u \in K_{\eta},
$$

which can be written as

$$
F(v)-F(u) \geq \log \left\{1+\left\langle F^{\prime}(u), \eta(v, u)\right\rangle\right\} \quad \forall v, u \in K_{\eta} .
$$

Changing the role of $u$ and $v$ in (3.4), we also have

$$
\left.F(u)-F(v) \geq \log \left\{1+\left\langle F^{\prime}(v), \eta(u, v)\right)\right\rangle,\right\} \quad \forall v, u \in K_{\eta} .
$$

Adding (3.4) and (3.5), we have

$$
\begin{aligned}
\left\langle F^{\prime}(u), \eta(v, u)\right\rangle+ & \left\langle F^{\prime}(v, \eta(u, v)\rangle\right. \\
& \left.\leq-\left(\left\langle F^{\prime}(u), \eta(v, u)\right\rangle\right)\left(F^{\prime}(v), \eta(u, v)\right\rangle\right),
\end{aligned}
$$

which express the $\eta$-monotonicity of the differential $F^{\prime}($.$) of the exponentially preinvex$ functions.

Theorem 3.7 enables us to introduce the concept of the exponentially $\eta$-monotone operators, which appears to be new.

Definition 3.1. The differential $F^{\prime}($.$) is said to be exponentially \eta$-mononotone, if

$$
\left\langle e^{F(u)} F^{\prime}(u), \eta(v, u)\right\rangle+\left\langle e^{F(v)} F^{\prime}(v), \eta(u, v)\right\rangle \leq 0, \quad \forall u, v \in H .
$$

Definition 3.2. The differential $F^{\prime}(\cdot)$ is said to be exponentially pseudo $\eta$-mononotone, if

$$
\left\langle e^{F(u)} F^{\prime}(u), \eta(v, u)\right\rangle \geq 0, \quad \Rightarrow-\left\langle e^{F(v)} F^{\prime}(v), \eta(v, u)\right\rangle \geq 0, \quad \forall u, v \in H .
$$

From these definitions, it follows that exponentially $\eta$-monotonicity implies exponentially pseudo $\eta$-monotonicity, but the converse is not true. 
Theorem 3.8. Let $F$ be differentiable exponentially preinvex function on the invex set $K_{\eta}$. and Condition $C$ hold. Then

$$
\left.e^{F(v)}-e^{F(u}\right) \geq\left\langle e^{F(u)} F^{\prime}(u), \eta(v, u)\right\rangle, \quad \forall v, u \in K_{\eta} .
$$

if and only if, $F^{\prime}$ satisfies

$$
\left\langle e^{F(u)} F^{\prime}(u), \eta(v, u)\right\rangle+\left\langle e^{F(v)} F^{\prime}(v), \eta(u, v)\right\rangle \leq 0, \quad \forall u, v \in K_{\eta} .
$$

Proof. Let $F$ be a exponentially preinvex function on the invex set $K_{\eta}$. Then, from Theorem 3.1, we have

$$
e^{F(v)}-e^{F(u)} \geq\left\langle e^{F(u)} F^{\prime}(u), \eta(v, u)\right\rangle, \quad \forall u, v \in K_{\eta} .
$$

Changing the role of $u$ and $v$ in (3.8), we have

$$
e^{F(u)}-e^{F(v)} \geq\left\langle e^{F(v)} F^{\prime}(v), \eta(u, v)\right\rangle, \quad \forall u, v \in K_{\eta} .
$$

Adding (3.8) and (3.9), we have

$$
\left\langle e^{F(u)} F^{\prime}(u), \eta(v, u)\right\rangle+\left\langle e^{F(v)} F^{\prime}(v), \eta(u, v)\right\rangle \leq 0, \quad \forall u, v \in K_{\eta},
$$

which shows that $F^{\prime}$ is exponentially $\eta$-monotone operator.

Conversely, from (3.7), we have

$$
\left.\left\langle e^{F(v)} F^{\prime}(v), \eta(u, v)\right\rangle \leq-\left\langle e^{F(u)} F^{\prime}(u), \eta(v, u)\right)\right\rangle .
$$

Since $K_{\eta}$ is an invex set, $\forall u, v \in K_{\eta}, \quad t \in[0,1] v_{t}=u+t \eta(v, u) \in K_{\eta}$. Taking $v=v_{t}$ in (3.10), we have

$$
\left\langle e^{F\left(v_{t}\right)} F^{\prime}\left(v_{t}\right), \eta\left(u, v_{t}\right)\right\rangle \leq\left\langle-e^{F(u)} F^{\prime}(u), \eta\left(v_{t}, u\right)\right\rangle,
$$

which implies, using the Condition $\mathrm{C}$, that

$$
\left\langle e^{F\left(v_{t}\right)} F^{\prime}\left(v_{t}\right), \eta(v, u)\right\rangle \geq\left\langle e^{F(u)} F^{\prime}(u), \eta(v, u)\right\rangle .
$$

Consider the auxiliary function

$$
g(t)=e^{F(u+t \eta(v, u))}
$$

from which, we have

$$
g(1)=e^{F(u+\eta(v, u))}, \quad g(0)=e^{F(u)} .
$$

Then, from (3.11), we have

$$
g^{\prime}(t)=\left\langle e^{F\left(v_{t}\right)} F^{\prime}\left(v_{t}\right), \eta(v, u)\right\rangle \geq\left\langle e^{F(u)} F^{\prime}(u), \eta(v, u)\right\rangle .
$$


Integrating (3.12) between 0 and 1 , we have

$$
g(1)-g(0)=\int_{0}^{1} g^{\prime}(t) d t \geq\left\langle e^{F(u)} F^{\prime}(u), \eta(v, u)\right\rangle .
$$

Thus it follows using the fact $e^{F(u+\eta(v, u))} \leq e^{F(v)}$, that

$$
e^{F(v)}-e^{F(u)} \geq\left\langle e^{F(u)} F^{\prime}(u), \eta(v, u)\right\rangle,
$$

which is the required (3.6).

We now give a necessary condition for exponentially pseudo-preinvex function.

Theorem 3.9. Let $F^{\prime}$ be exponentially pseudomonotone. Then $F$ is a exponentially pseudo-invex function.

Proof. Let $F^{\prime}$ be a exponentially pseudomonotone operator. Then, $\forall u, v \in K_{\eta}$,

$$
\left\langle e^{F(u)} F^{\prime}(u), \eta(v, u)\right\rangle \geq 0 .
$$

implies that

$$
-\left\langle e^{F(v)} F^{\prime}(v), \eta(v, u)\right\rangle \geq 0 .
$$

Since $K$ is an invex set, $\forall u, v \in K_{\eta}, t \in[0,1], v_{t}=u+t \eta(v, u) \in K_{\eta}$. Taking $v=v_{t}$ in (3.13), we have

$$
\left\langle e^{F\left(v_{t}\right)} F^{\prime}\left(v_{t}\right), \eta(v, u)\right\rangle \geq 0 .
$$

Consider the auxiliary function

$$
g(t)=e^{F(u+t \eta(v, u))}=e^{F\left(v_{t}\right)}, \quad \forall u, v \in K_{\eta}, t \in[0,1],
$$

which is differentiable, since $F$ is differentiable function. Then, using (3.14), we have

$$
g^{\prime}(t)=\left\langle e^{F\left(v_{t}\right)} F^{\prime}\left(v_{t}\right), \eta(v, u)\right\rangle \geq 0 .
$$

Integrating the above relation between 0 to 1 , we have

$$
g(1)-g(0)=\int_{0}^{1} g^{\prime}(t) d t \geq 0,
$$

that is,

$$
e^{F(v)}-e^{F(u)} \geq e^{F(u+\eta(v, u))}-e^{F(u)} \geq 0,
$$

showing that $F$ is a exponentially pseudo-invex function. 
Definition 3.3. The function $F$ is said to be sharply exponentially pseudo invex, if

$$
\begin{aligned}
\left\langle e^{F(u)} F^{\prime}(u), \eta(v, u)\right\rangle & \geq 0 \\
& \Rightarrow \\
F(v) & \geq e^{F(v+t(u-v))}, \quad \forall u, v \in K_{\eta}, t \in[0,1] .
\end{aligned}
$$

Theorem 3.10. Let $F$ be a sharply exponentially pseudo invex function on $K$. Then

$$
\left\langle e^{F(v)} F^{\prime}(v), v-u\right\rangle \geq 0, \quad \forall u, v \in K_{\eta} .
$$

Proof. Let $F$ be a sharply exponentially pesudo invex function on $K$. Then

$$
e^{F(v)} \geq e^{F(v+t \eta(u, v))}, \quad \forall u, v \in K_{\eta}, t \in[0,1] .
$$

from which we have

$$
0 \leq \lim _{t \rightarrow 0}\left\{\frac{e^{F(v+t \eta(v, u)))}-e^{F(v)}}{t}\right\}=\left\langle e^{F(v)} F^{\prime}(v), \eta(v, u)\right\rangle,
$$

the required result.

Definition 3.4. A function $F$ is said to be a exponentially pseudo preinvex function, if there exists a strictly positive bifunction $b(.,$.$) , such that$

$$
\begin{aligned}
e^{F(v)} & <e^{F(u)} \\
& \Rightarrow \\
\eta(v, u)) & <e^{F(u)}+t(t-1) b(v, u), \forall u, v \in K_{\eta}, t \in[0,1] .
\end{aligned}
$$

Theorem 3.11. If the function $F$ is an exponentially preinvex function such that $e^{F(v)}<e^{F(u)}$, then the function $F$ is exponentially pseudo preinvex.

Proof. Since $e^{F(v)}<e^{F(u)}$ and $F$ is exponentially preinvex function, then $\forall u, v \in$ $K_{\eta}, \quad t \in[0,1]$, we have

$$
\begin{aligned}
e^{F(u+t \eta(v, u)))} & \leq e^{F(u)}+t\left(e^{F(v)}-e^{F(u)}\right) \\
& <e^{F(u)}+t(1-t)\left(e^{F(v)}-e^{F(u}\right) \\
& \left.=e^{F(u)}+t(t-1)\left(e^{F(u)}-e^{F(v)}\right)\right) \\
& <e^{F(u)}+t(t-1) b(u, v),
\end{aligned}
$$

where $b(u, v)=e^{F(u)}-e^{F(v)}>0$, the required result. This shows that the function $F$ is an exponentially pseudo preinvex function.

We now discuss the optimality condition for the differentiable exponentially preinvex functions, which is the main motivation of our next result. 
Theorem 3.12. Let $F$ be a differentiable exponentially preinvex function. Then $u \in K_{\eta}$ is the minimum of the function $F$, if and only if, $u \in K_{\eta}$ satisfies the inequality

$$
\left\langle e^{F(u)} F^{\prime}(u), \eta(v, u)\right\rangle \geq 0, \quad \forall u, v \in K_{\eta} .
$$

which is called the exponentially variational-like inequality.

Proof. Let $u \in K_{\eta}$ be a minimum of the function $F$. Then

$$
F(u) \leq F(v), \forall v \in K_{\eta} .
$$

from which, we have

$$
e^{F(u)} \leq e^{F(v)}, \forall v \in K_{\eta} .
$$

Since $K_{\eta}$ is a convex set, so, $\forall u, v \in K_{\eta}, \quad t \in[0,1]$,

$$
v_{t}=u+t \eta(v, u) \in K_{\eta} .
$$

Taking $v=v_{t}$ in (3.16), we have

$$
0 \leq \lim _{t \rightarrow 0}\left\{\frac{e^{F(u+t \eta(v, u))}-e^{F(u)}}{t}\right\}=\left\langle e^{F(u)} F^{\prime}(u), \eta(v, u)\right\rangle .
$$

Since $F$ is differentiable exponentially preinvex function, so

$$
e^{F(u+t \eta(v, u))} \leq e^{F(u)}+t\left(e^{F(v)}-e^{F(u)}\right), \quad u, v \in K_{\eta}, t \in[0,1],
$$

from which, using (3.17), we have

$$
e^{F(v)}-e^{F(u)} \geq \lim _{t \rightarrow 0}\left\{\frac{e^{F(u+t \eta(v, u))}-e^{F(u)}}{t}\right\}=\left\langle e^{F(u)} F^{\prime}(u), \eta(v, u)\right\rangle \geq 0,
$$

from which, we have

$$
e^{F(v)}-e^{F(u)} \geq 0
$$

which implies that

$$
F(u) \leq F(v), \quad \forall v \in K_{\eta} .
$$

This shows that $u \in K_{\eta}$ is the minimum of the differentiable exponentialy preinvex function the required result.

Remark 3.2. The inequality of the type (3.15) is called the exponentiall variational-like inequality, which has been introduced and studied by Noor [21].

We now show that the difference of exponentially preinvex functions and exponentially affine preinvex functions is again an exponentially preinvex function. 
Theorem 3.13. $\quad$ Let $f$ be a exponentially affine preinvex function. Then $F$ is a exponentially preinvex function, if and only if, $H=F-$ is a exponentially preivex function.

Proof. Let $f$ be exponentially affine preinvex function. Then

$$
e^{f(u+t \eta(v, u))}=(1-t) e^{f(u)}+t e^{f(v)}, \quad \forall u, v \in K_{\eta}, \quad t \in[0,1] .
$$

From the exponentially preinvexity of $F$, we have

$$
e^{F(u+t \eta(v, u))} \leq(1-t) e^{F(u)}+t e^{F(v)}, \quad \forall u, v \in K_{\eta}, \quad t \in[0,1] .
$$

From (3.18) and (3.19), we have

$$
\begin{aligned}
e^{F((u+t \eta(v, u))} & -e^{f((u+t \eta(v, u)} \\
& \leq(1-t)\left(e^{F(u)}-e^{f(g(u))}\right)+t\left(e^{F(v)}-e^{f(v)}\right),
\end{aligned}
$$

from which, it follows that

$$
\begin{aligned}
e^{H((u+t \eta(v, u)} & =e^{F((u+t \eta(v, u))}-e^{f((1-t) f(u+t \eta(v \cdot u)} \\
& \leq(1-t)\left(e^{F(u)}-e^{f(u)}\right)+t\left(e^{F(v)}-e^{f(v)}\right),
\end{aligned}
$$

which show that $H=F-f$ is an exponentially preinvex function.

The inverse implication is obvious.

We would like to remark that one can show that a function $F$ is a exponentially preinvex function, if and only if, $F$ is exponentially affine preinvex function. It is worth mentioning that the exponentially preinvex function is also a Wright exponentially preinvex function. From the definition 2.4, we have

$$
e^{F(u+t \eta(v, u))}+e^{F(v+t \eta(u, v)} \leq e^{F}(u)+e^{F}(v), \forall u, v \in K_{\eta}, t \in[0,1],
$$

which is called Wright exponentially preinvex function. It is an interesting problem to study the properties and applications of the Wright exponentially preinvex functions.

\section{Conclusion}

In this paper, we have introduced and studied a new class of preinvex functions which is called the exponentially preinvex function. It has been shown that exponentially preinvex functions enjoy several properties which convex functions have. We have shown that the minimum of the expedientially differentiable preinvex functions can be characterized by a new class of variational inequalities, which is called the exponential variational-like inequality. Several new properties of the exponentially preinvex functions have been studied. Exponentially variational-like inequalities is new one, which will be the subject of further research. This may stimulate further research. 


\section{Acknowledgements}

The authors would like to thank the Rector, COMSATS University Islamabad, Pakistan, for providing excellent research and academic environments.

\section{REF E R E N C E S}

1. G. Alirezaei and R. Mazhar, On exponentially concave functions and their impact in information theory, J. Inform. Theory Appl. 9(5)(2018), 265-275.

2. T. Antczak, On (p,r)-invex sets and functions, J. Math. Anal. Appl,263(2001), 355379 .

3. M. Avriel, $r$-Convex functions. Math. Program., 2(1972), 309-323.

4. M. U. Awan, M. A. Noor and K. I. Noor,Hermite-Hadamard inequalities for exponentially convex functions, 12(2)(2018), 405-409.

5. A. Ben-Israel and B. Mond, What is invexity?, J. Aust. Math. Soc. Ser. B 28 (1986) $1-9$.

6. S. N. Bernstein, Sur les fonctions absolument monotones, Acta Math. 52 (1929), 1-66.

7. G. Cristescu and L. Lupsa, Non Connected Convexities and Applications, Kluwer Academic Publisher, Dordrechet, (2002).

8. S. S. Dragomir, Inequalities of Hermite-Hadamard type for $\varphi$-convex functions, Konuralp J. Math.4 (1)(2016),54 - 67.

9. S. S. Dragomir and I. Gomm, Some Hermite-Hadamard type inequalities for functions whose exponentials are convex, Stud. Univ. Babes-Bolyai Math, 60(4)(2015), 527-534.

10. S. S. Dragomir and I. Gomm, Some Hermite-Hadamard type inequalities for functions whose product with exponentials are convex, Univ. Bacau. Sci. Stu. Res. Ser. Math. Info, 1(25)(2015), 145-158.

11. J. Hadamard, Etude sur les proprietes des fonctions entieres e. $t$ en particulier dune fonction consideree par Riemann, J. Math. Pure. Appl, 58(1893), 171-215.

12. M. A. Hanson, On sufficiency of the Kuhn-Tucker conditions, J. Math. Anal. Appl., 80 (1981) $545-550$.

13. C. Hermite, Sur deux limites d'une integrale definie, Mathesis, 3(82)(1883).

14. S. R. Mohan and S. K. Neogy, On invex sets and preinvex functions, J. Math. Anal. Appl., 189(1995), 901-908.

15. C. P. Niculescu and L. E. Persson, Convex Functions and Their Applications, SpringerVerlag, New York, (2018).

16. M. A. Noor, Variational like inequalities, Optimization, 30 (1994), 323-33

17. M. A. Noor, Fuzzy preinvex functions, Fuzzy Sets and Systems, 64 (1994), 95-104.

18. M. A. Noor, Hermite-Hadamard integral inequalities for log-preinvex functions, J. Math. Anal. Approx. Theory, 2 (2007), 126-131.

19. M. A. Noor, On Hermite-Hadamard integral inequalities for involving two log-preinvex functions, J. Inequal. Pure and Appl. Math., 3 (2007), 75-81.

20. M. A. Noor, New approximation schemes for generalvariational inequalities, J. Math. Anal. Appl.,251(2000), 217-229. 
21. M. A. Noor, Variational Inequalities and Optimization, Lecture Notes, Mathematics Department, COMSATS University Islamabad, Pakistan, (2008-2019).

22. M. A. Noor, Invex equilibrium problems, J. Math. Anal. Appl. 302(2005),463-475.

23. M. A. Noor, On generalized preinvex functions and monontonicities, J. Inequal. Pure Appl Math. 5(4)(2004), Article: 110.

24. M. A. Noor and K. I. Noor, Some charaxterizations of strongly preinvex functions, J. Math. Anal. Appl. 316(2)(2006), 697-706.

25. M. A. Noor and K. I. Noor, Exponentially convex functions, J. Orisa Math. Soc., 39(2019).

26. M. A. Noor and K. I. Noor, Strongly exponentially convex functions, U.P.B. Bull Sci. Appl. Math. Series A., 81(2019), xx-xx.

27. M. A. Noor and K. I. Noor, Strongly exponentially convex functions and their properties, J. Advanc. Math. Studies, 9(2)(2019). 180-188.

28. M. A. Noor and K. I. Noor, Some properties of differentiable exponential convex functions, preprint, (2019).

29. J. Pecaric, F. Proschan and Y. L. Tong, Convex Functions, Partial Orderings and Statistical applications, Academic Press, New York, (1992).

30. J. Pecaric, C. E. M. Pearce and V. Simic, Stolarsky means and Hadamard's inequality, J. Math. Anal. Appl, 220(1998), 99-109.

31. J. Pecaric and J. Jaksetic, On exponential convexity, Euler-Radau expansions and stolarsky means, Rad Hrvat. Matematicke Znanosti, 17(515)(2013), 81-94.

32. S. Pal and T. K. L. Wong, On exponentially concave functions and a new information geometry, Annals. Prob. 46(2)(2018), 1070-1113.

33. T. Weir and B. Mond, Preinvex functions in multiobjective optimization, J. Math. Anal. Appl., 136 (1988), 29-38.

\author{
Muhammad Aslam Noor \\ Department of Mathematics \\ COMSATS University Islamabad \\ Islamabad, Pakistan \\ noormaslam@gmail.com \\ Khalida Inayat Noor \\ Department of Mathematics \\ COMSATS University Islamabad \\ Islamabad, Pakistan \\ khalidan@gmail.com
}

